\title{
A CYCLIC THERMAL INSTABILITY IN CONTACT
}

\section{B I N A R Y S T A R S*}

\author{
B R I A N P. F L A N NER Y** \\ Institute for Advanced Study, Princeton, N.J. 08540, U.S.A.
}

\begin{abstract}
Contact binary stars coupled by a common convective envelope in which the entropy is constant, the Lucy model, are unstable against mass exchange: if either component begins to transfer mass, it will continue to do so. A detailed sequence of models is calculated which follows the thermal evolution of a $2 M_{\odot}$ contact binary of normal Population I abundances $(X=0.70, Z=0.02)$, starting at nearly equal mass. The initial instability develops into a cyclic mass-exchange with the mass fraction oscillating between $0.56 \leqslant m_{2} /\left(m_{1}+m_{2}\right) \leqslant 0.62$ with a period of $\sim 10^{7} \mathrm{yr}$. Throughout the cycle the component stars are not in thermal equilibrium. The instability is of a general nature, and such oscillating systems can satisfactorily populate the short period, red region of the period color relation for W UMa stars.
\end{abstract}

\footnotetext{
* A detailed account of this study has been published in the Astrophysical Journal.

** Research sponsored by the National Science Foundation: Grant No. NSF GP 40768X.
} 